\title{
Silicon sensor probing and radiation studies for the LHCb Silicon Tracker
}

\author{
Cristina Lois Gómez \\ Physik-Institut der Universität Zürich \\ Universidade de Santiago de Compostela \\ lois@physik.unizh.ch
}




\section{LHCb Silicon Tracker group}

On behalf of LHCb Silicon Tracker Group, $\approx 50$ researchers from 6 institutes:

- Max-Planck-Institut für Kernphysik, Heidelberg

- Kiev Institute for Nuclear Research

- Laboratoire de Physique des Hautes Energies, Lausanne

- Budker Institute for Nuclear Physics, Novosibirsk

- Universidade de Santiago de Compostela

- Physik-Institut der Universität Zürich 


\section{Overview}

- LHCb spectrometer and the Silicon Tracker 


\section{Overview}

- LHCb spectrometer and the Silicon Tracker

- Sensor quality assurance program and results on first sensors 


\section{Overview}

- LHCb spectrometer and the Silicon Tracker

- Sensor quality assurance program and results on first sensors

- Irradiation tests with IT prototype sensors 


\section{Overview}

- LHCb spectrometer and the Silicon Tracker

- Sensor quality assurance program and results on first sensors

- Irradiation tests with IT prototype sensors

- Summary 


\section{Overview}

- LHCb spectrometer and the Silicon Tracker

- Sensor quality assurance program and results on first sensors

- Irradiation tests with IT prototype sensors

- Summary 


\section{Overview}

- LHCb spectrometer and the Silicon Tracker

- Sensor quality assurance program and results on first sensors

- Irradiation tests with IT prototype sensors

- Summary 


\section{LHCb spectrometer}

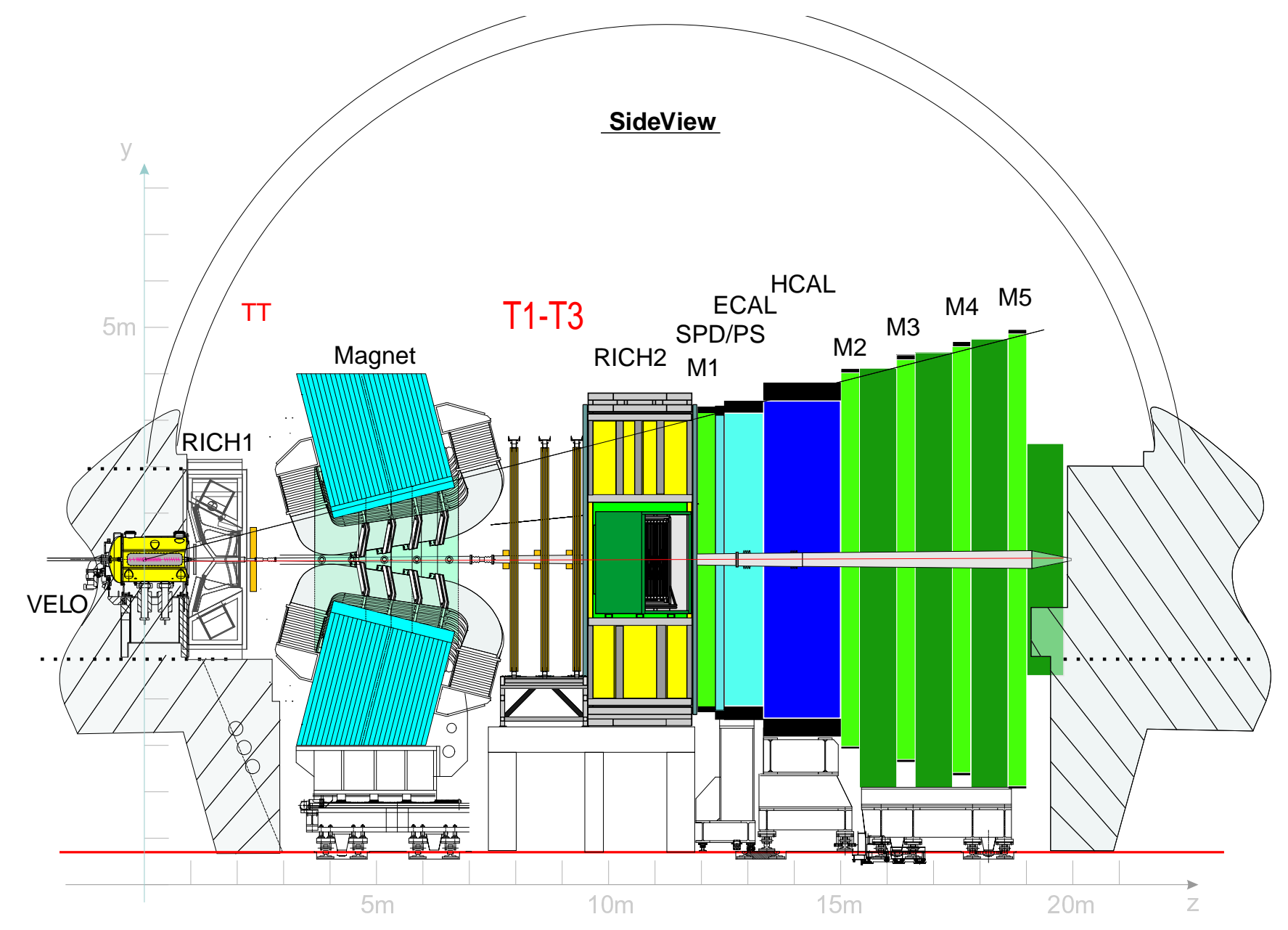




\section{Trigger Tracker}

- 4 detection layers

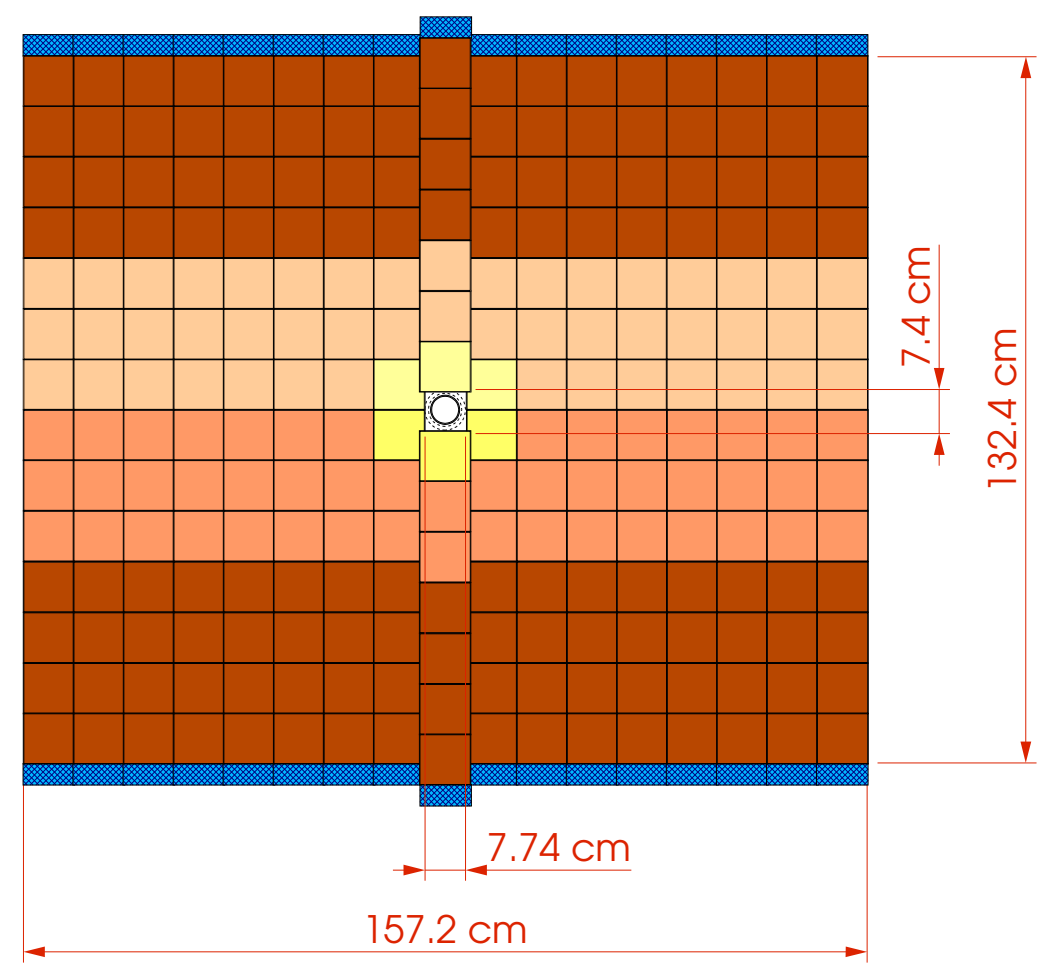




\section{Trigger Tracker}

- 4 detection layers

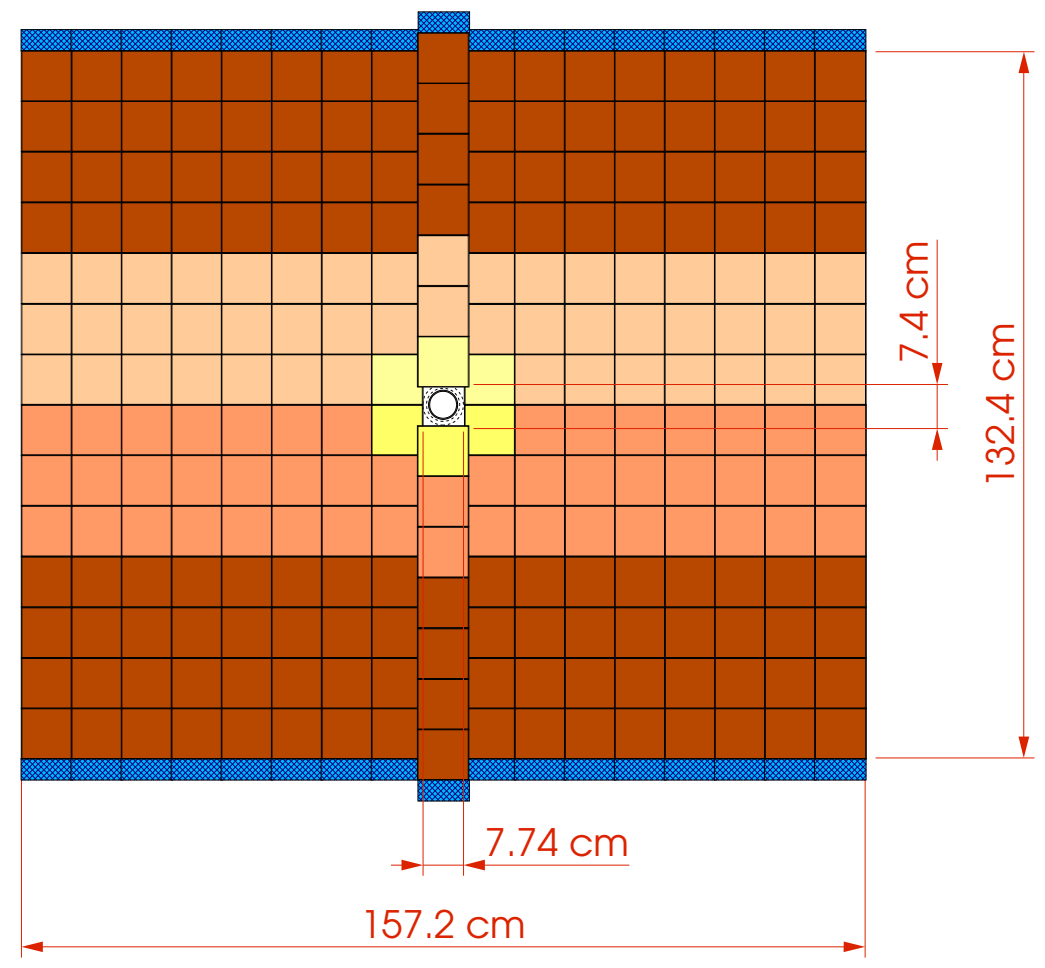

- 14- and 7-sensor modules, several readout sectors 


\section{Trigger Tracker}
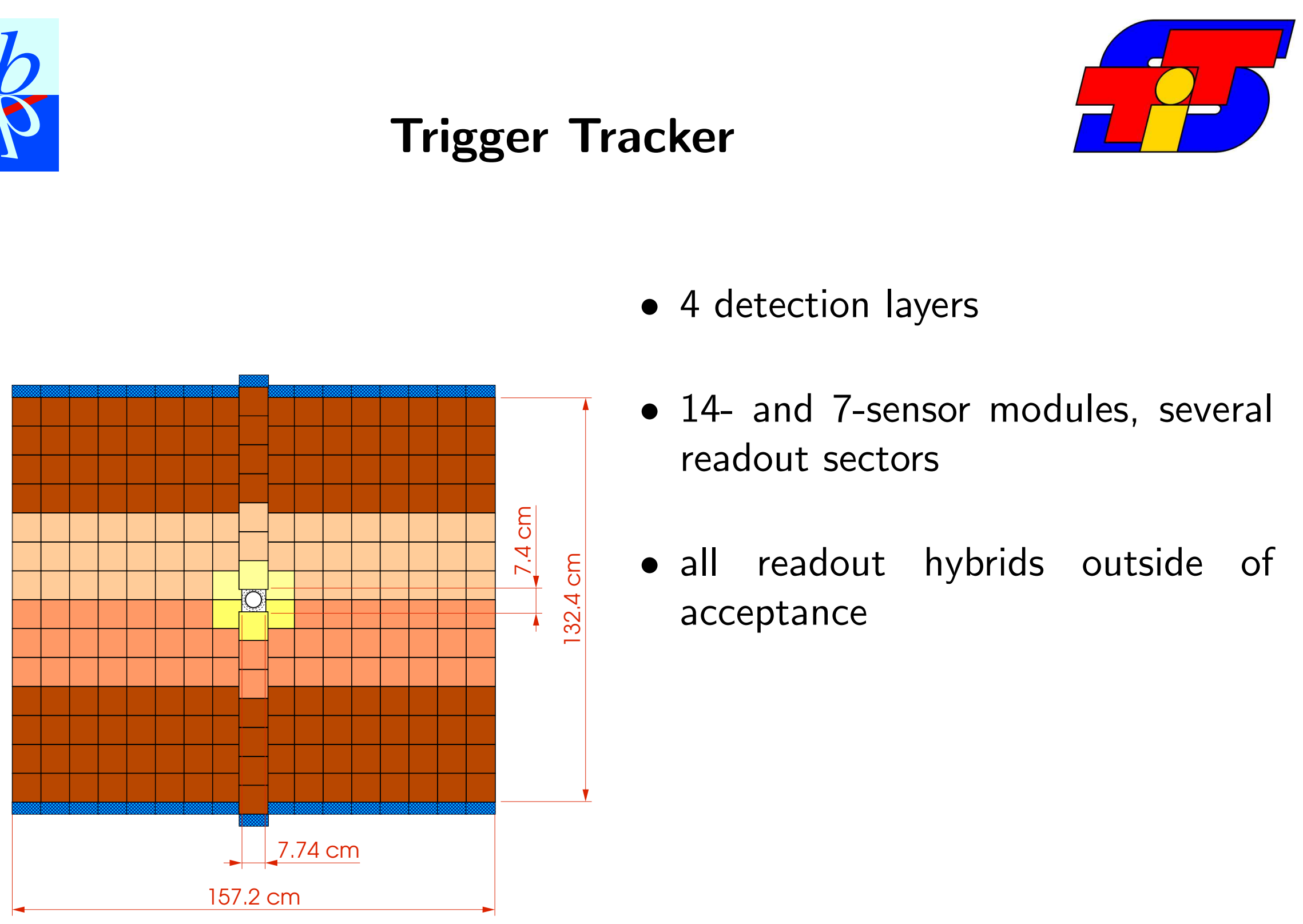

- 4 detection layers

- 14- and 7-sensor modules, several readout sectors

- all readout hybrids outside of acceptance 


\section{Trigger Tracker}

- 4 detection layers

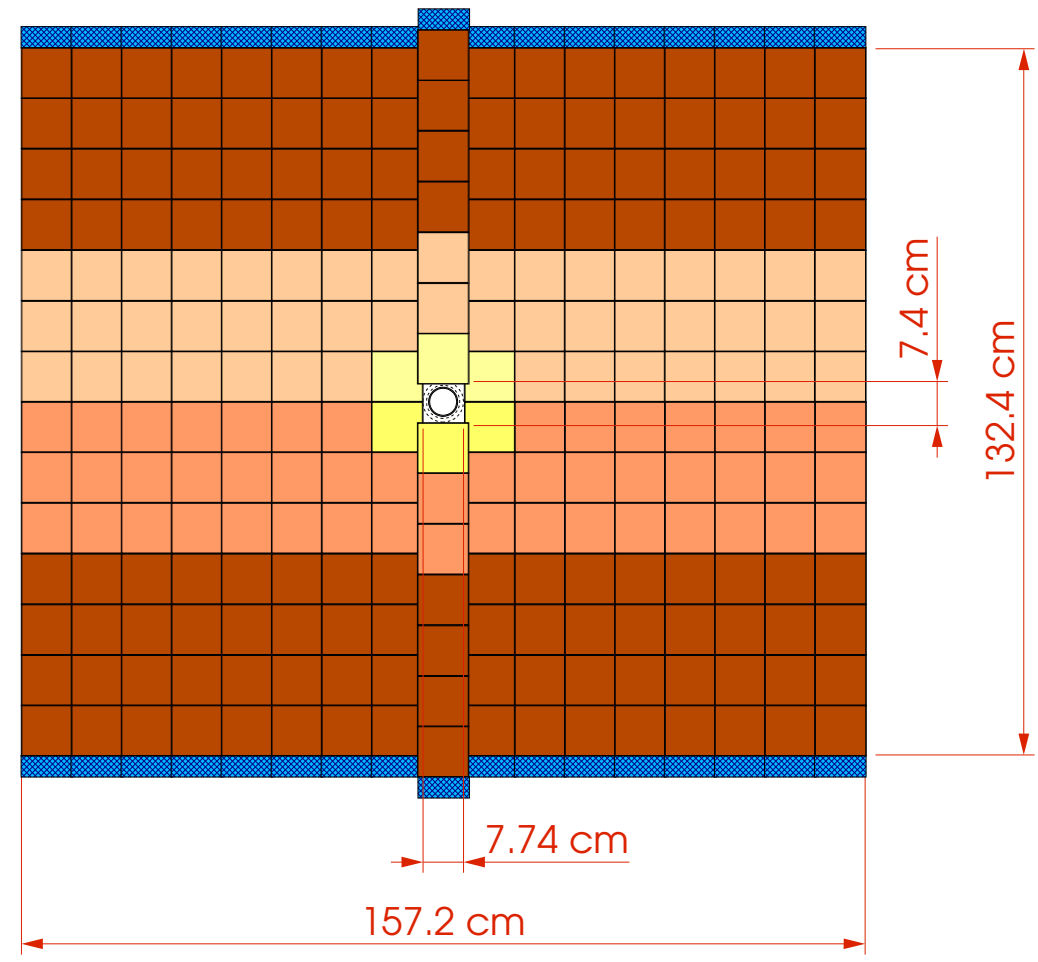

- 14- and 7-sensor modules, several readout sectors

- all readout hybrids outside of acceptance

- silicon microstrip detectors type CMS-OB2 


\section{Trigger Tracker}

- 4 detection layers

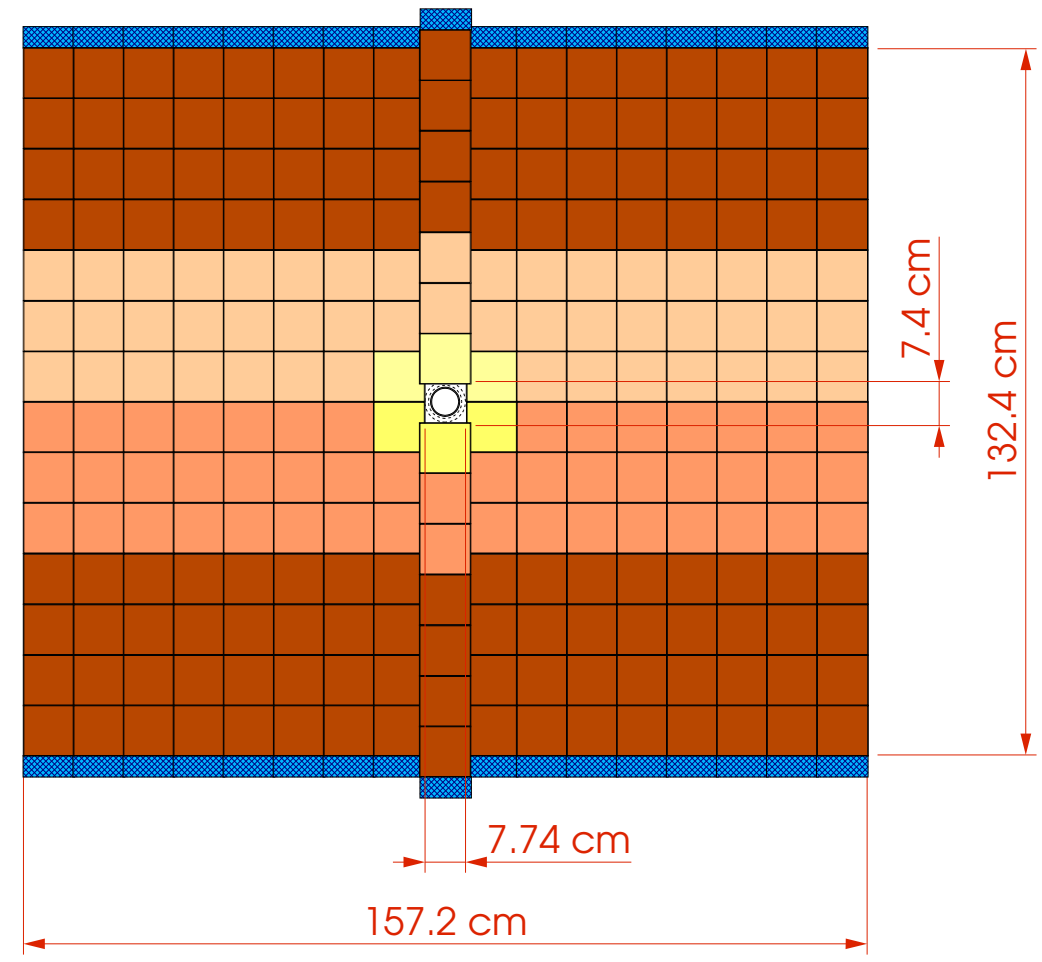

- 14- and 7-sensor modules, several readout sectors

- all readout hybrids outside of acceptance

- silicon microstrip detectors type CMS-OB2

- $500 \mu \mathrm{m}$ thick

- $\mathrm{p}$-on- $\mathrm{n}$, single-sided

- $183 \mu \mathrm{m}$ pitch, $w / p=0.25$

- dimensions $9.4 \times 9.6 \mathrm{~cm}^{2}$ 


\section{Inner Tracker}

- 3 stations (T1-T3)

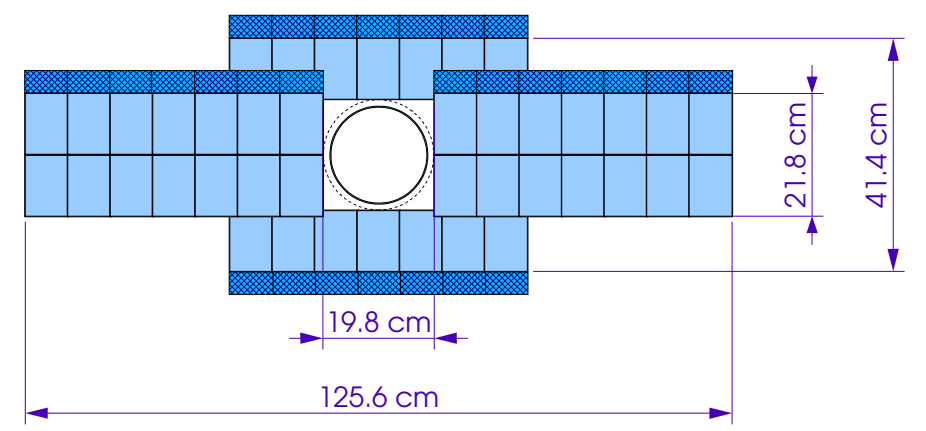




\section{Inner Tracker}

- 3 stations (T1-T3)

- 4 detection layers per station

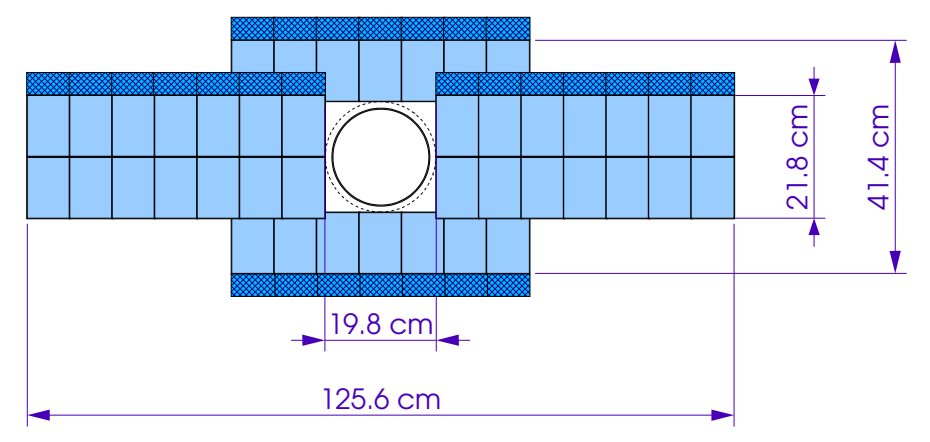




\section{Inner Tracker}

- 3 stations (T1-T3)

- 4 detection layers per station

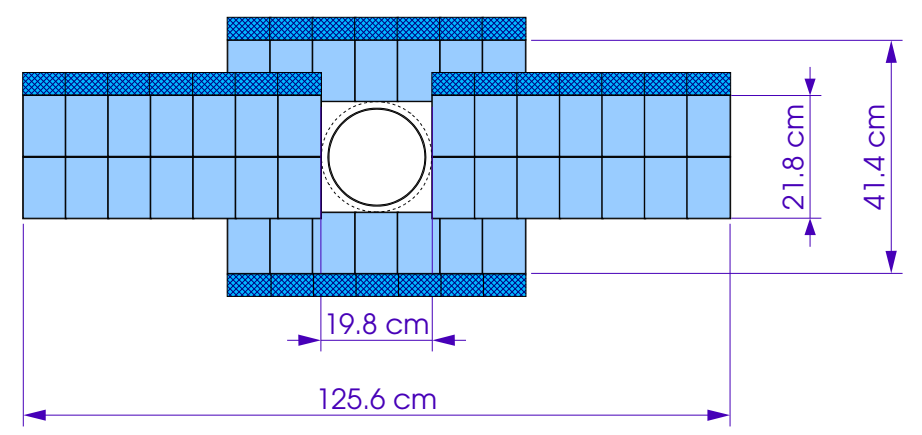

- 4 individual boxes per station 


\section{Inner Tracker}

- 3 stations (T1-T3)

- 4 detection layers per station

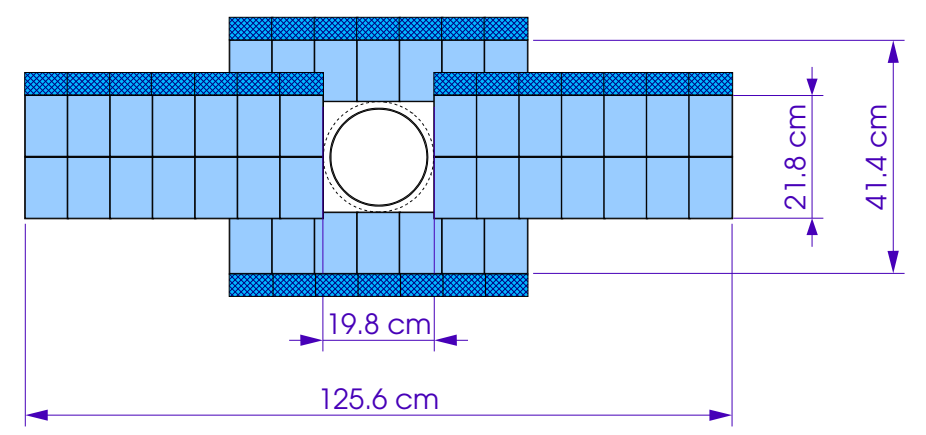

- 4 individual boxes per station

- 1- and 2-sensor modules 


\section{Inner Tracker}

- 3 stations (T1-T3)

- 4 detection layers per station

- 4 individual boxes per station

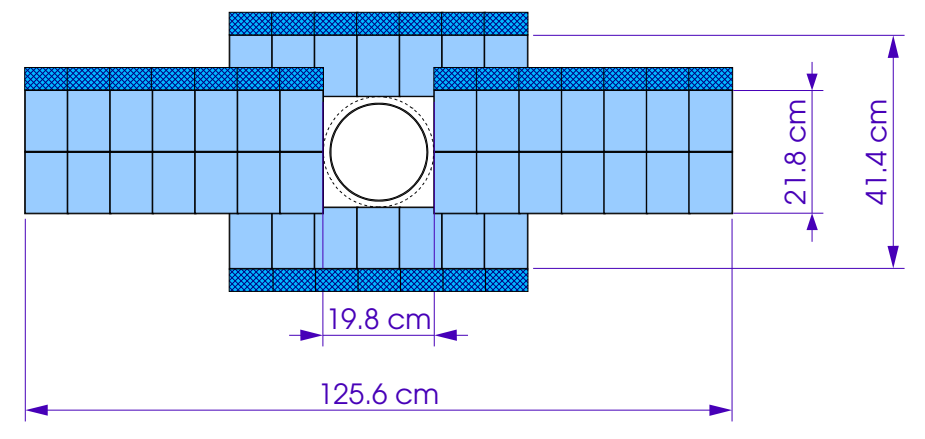

- 1- and 2-sensor modules

- silicon microstrip detectors (HPK320, HPK-410) 


\section{Inner Tracker}

- 3 stations (T1-T3)

- 4 detection layers per station

- 4 individual boxes per station

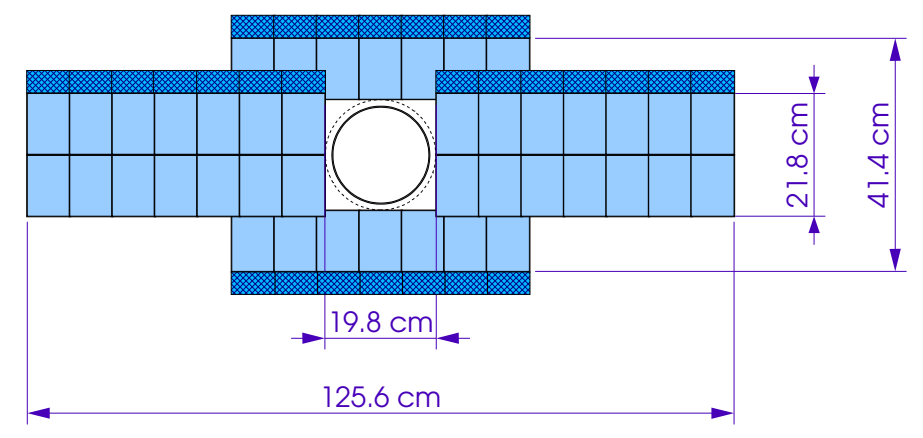

- 1- and 2-sensor modules

- silicon microstrip detectors (HPK320, HPK-410)

- $320 \mu \mathrm{m} / 410 \mu \mathrm{m}$ thick

- p-on-n, single-sided

- $198 \mu \mathrm{m}$ pitch, $w / p=0.25$

- dimensions $11 \times 7.8 \mathrm{~cm}^{2}$ 


\section{Overview}

- LHCb spectrometer and the Silicon Tracker

- Sensor quality assurance program and results on first sensors

- Irradiation tests with IT prototype sensors

- Summary 


\section{Sensor quality assurance program}

- Required to ensure excellent performance of modules 


\section{Sensor quality assurance program}

- Required to ensure excellent performance of modules

- Results for first batches of sensors 


\section{Sensor quality assurance program}

- Required to ensure excellent performance of modules

- Results for first batches of sensors

\begin{tabular}{lcc} 
Sensor type & Delivered & Total production \\
\hline HPK-320 & 14 & 194 \\
\hline HPK-410 & 35 & 386 \\
\hline HPK-500 & 98 & 1000 \\
\hline
\end{tabular}




\section{Sensor quality assurance program}

- Required to ensure excellent performance of modules

- Results for first batches of sensors

\begin{tabular}{lcc} 
Sensor type & Delivered & Total production \\
\hline HPK-320 & 14 & 194 \\
\hline HPK-410 & 35 & 386 \\
\hline HPK-500 & 98 & 1000 \\
\hline
\end{tabular}

- Two stages: 


\section{Sensor quality assurance program}

- Required to ensure excellent performance of modules

- Results for first batches of sensors

\begin{tabular}{lcc} 
Sensor type & Delivered & Total production \\
\hline HPK-320 & 14 & 194 \\
\hline HPK-410 & 35 & 386 \\
\hline HPK-500 & 98 & 1000 \\
\hline
\end{tabular}

- Two stages:

- tests performed by HPK prior to shipment 


\section{Sensor quality assurance program}

- Required to ensure excellent performance of modules

- Results for first batches of sensors

\begin{tabular}{lcc} 
Sensor type & Delivered & Total production \\
\hline HPK-320 & 14 & 194 \\
\hline HPK-410 & 35 & 386 \\
\hline HPK-500 & 98 & 1000 \\
\hline
\end{tabular}

- Two stages:

- tests performed by HPK prior to shipment

- tests performed by our group after reception 


\section{Tests performed by the ST group}




\section{Tests performed by the ST group}

- Visual inspection 


\section{Tests performed by the ST group}

- Visual inspection

- IV curves 


\section{Tests performed by the ST group}

- Visual inspection

- IV curves

- CV curves 


\section{Tests performed by the ST group}

- Visual inspection

- IV curves

- CV curves

- Metrological measurements 


\section{Tests performed by the ST group}

- Visual inspection

- IV curves

- CV curves

- Metrological measurements

- Other tests 


\section{Tests performed by the ST group}

- Visual inspection

- IV curves

- CV curves

- Metrological measurements

- Other tests 


\section{Visual inspection}

- Examine sensors under microscope:

- take note of scratches/defects

- look for chipped edges

- look for pad bondability/contamination

- check serial \# on scratch-pad 


\section{Visual inspection}

- Examine sensors under microscope:

- take note of scratches/defects

- look for chipped edges

- look for pad bondability/contamination

- check serial \# on scratch-pad

- sensors very good: no deep scratches or big defects found 


\section{Leakage Currents}

- IV curves taken up to $500 \mathrm{~V}$, at $\mathrm{T} \sim 20{ }^{\circ} \mathrm{C}, \mathrm{RH}<30 \%$

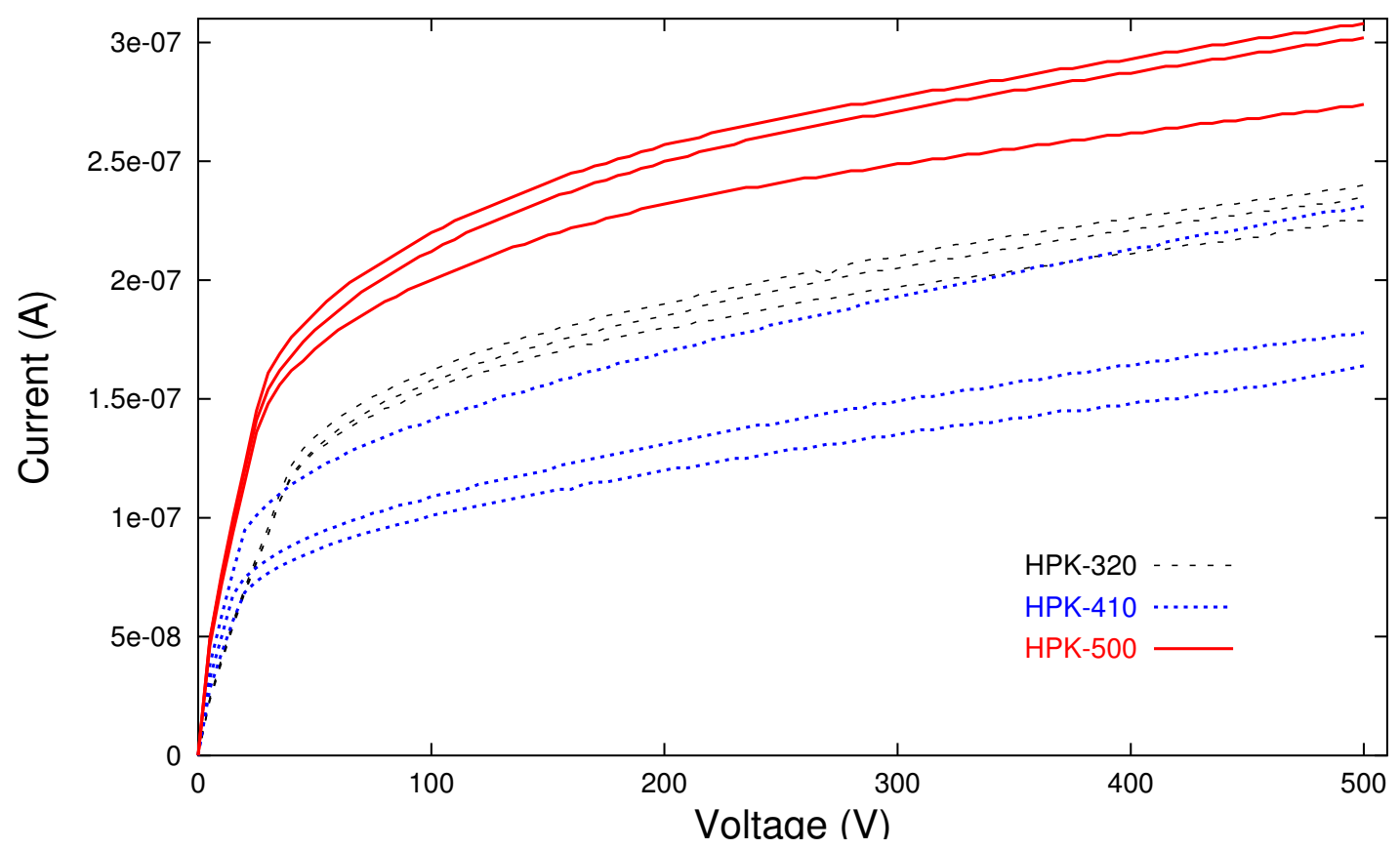




\section{Leakage Currents}

- IV curves taken up to $500 \mathrm{~V}$, at $\mathrm{T} \sim 20{ }^{\circ} \mathrm{C}, \mathrm{RH}<30 \%$

- Great uniformity in currents; no breakdown below $500 \mathrm{~V}$

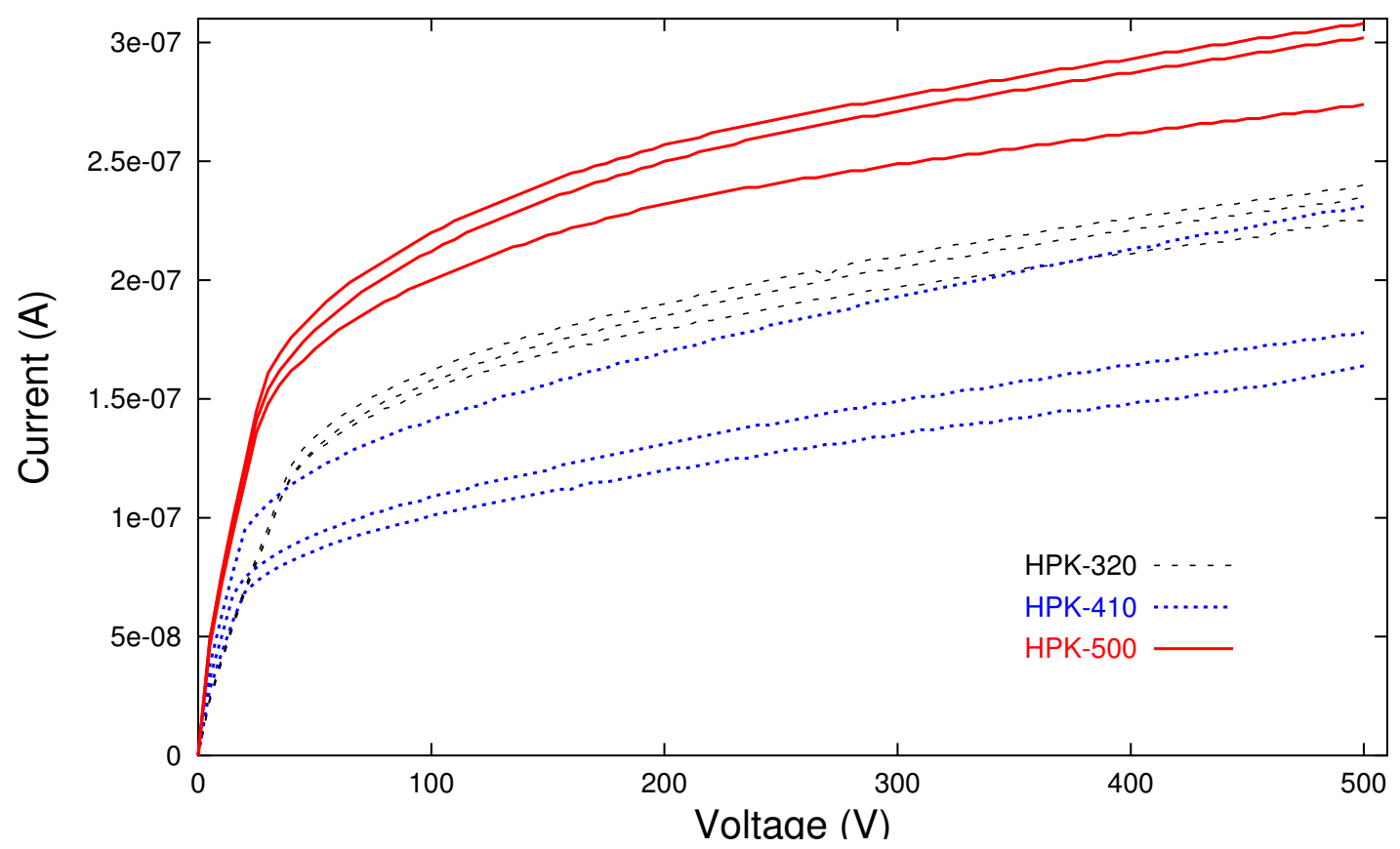




\section{Leakage Currents}

- Observed low currents; typically $I<400 \mathrm{nA}$ at $500 \mathrm{~V}$

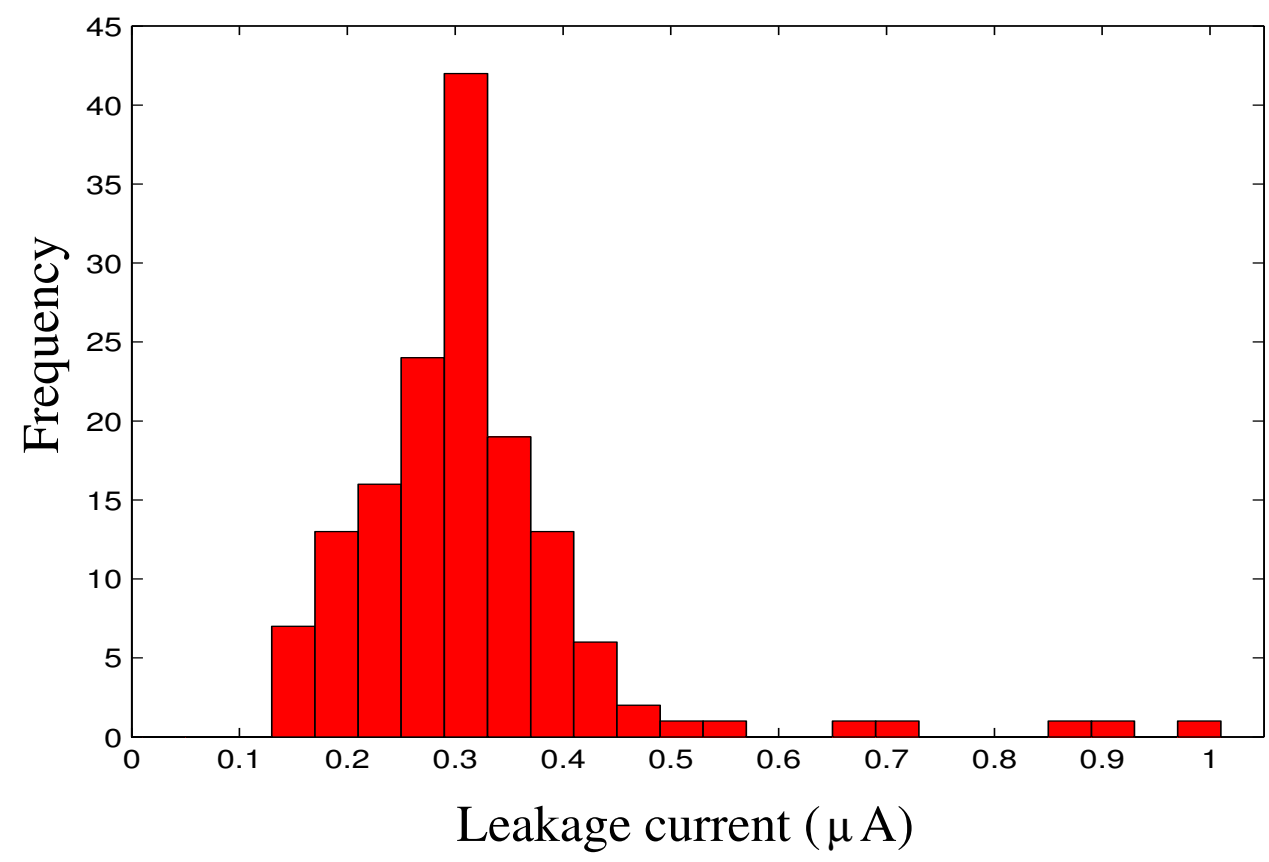




\section{Leakage Currents}

- Observed low currents; typically $I<400 \mathrm{nA}$ at $500 \mathrm{~V}$

- All sensors fulfill specifications

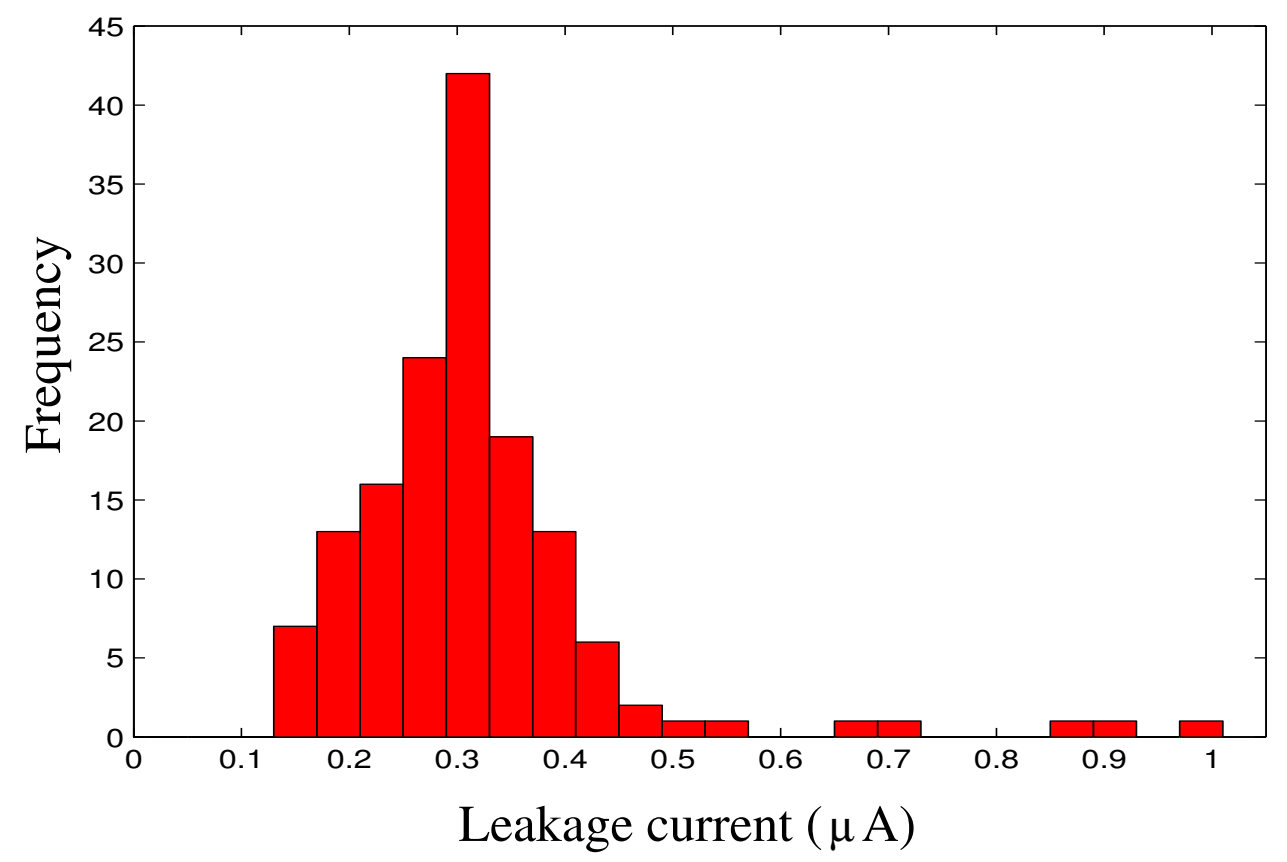




\section{Full depletion voltage}

- $\mathrm{CV}$ curves taken at $\mathrm{T} \sim 20{ }^{\circ} \mathrm{C}, \mathrm{RH}<30 \%, f=1 \mathrm{kHz}$ 


\section{Full depletion voltage}

- $\mathrm{CV}$ curves taken at $\mathrm{T} \sim 20{ }^{\circ} \mathrm{C}, \mathrm{RH}<30 \%, f=1 \mathrm{kHz}$

- All sensors fulfill specifications

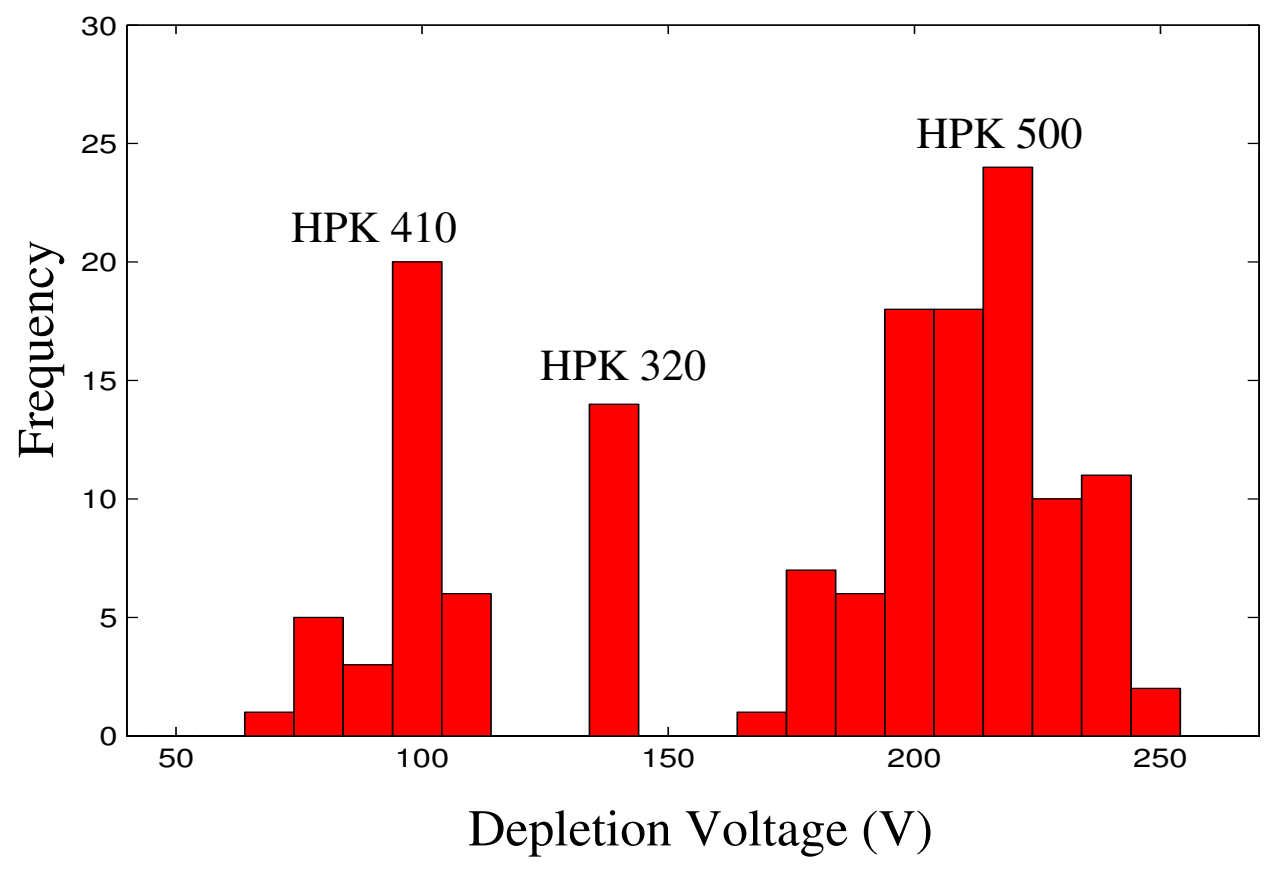

10th European Symposium on Semiconductor Detectors 


\section{Metrology measurements}

- optical metrology machine Mahr OMS 600 


\section{Metrology measurements}

- optical metrology machine Mahr OMS 600

- flatness, length, width, parallelity, etc. 


\section{Metrology measurements}

- optical metrology machine Mahr OMS 600

- flatness, length, width, parallelity, etc.

- All sensors very good 


\section{Metrology measurements}

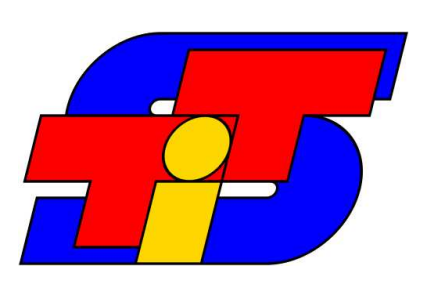

- optical metrology machine Mahr OMS 600

- flatness, length, width, parallelity, etc.

- All sensors very good

Warp Profile HPK 320

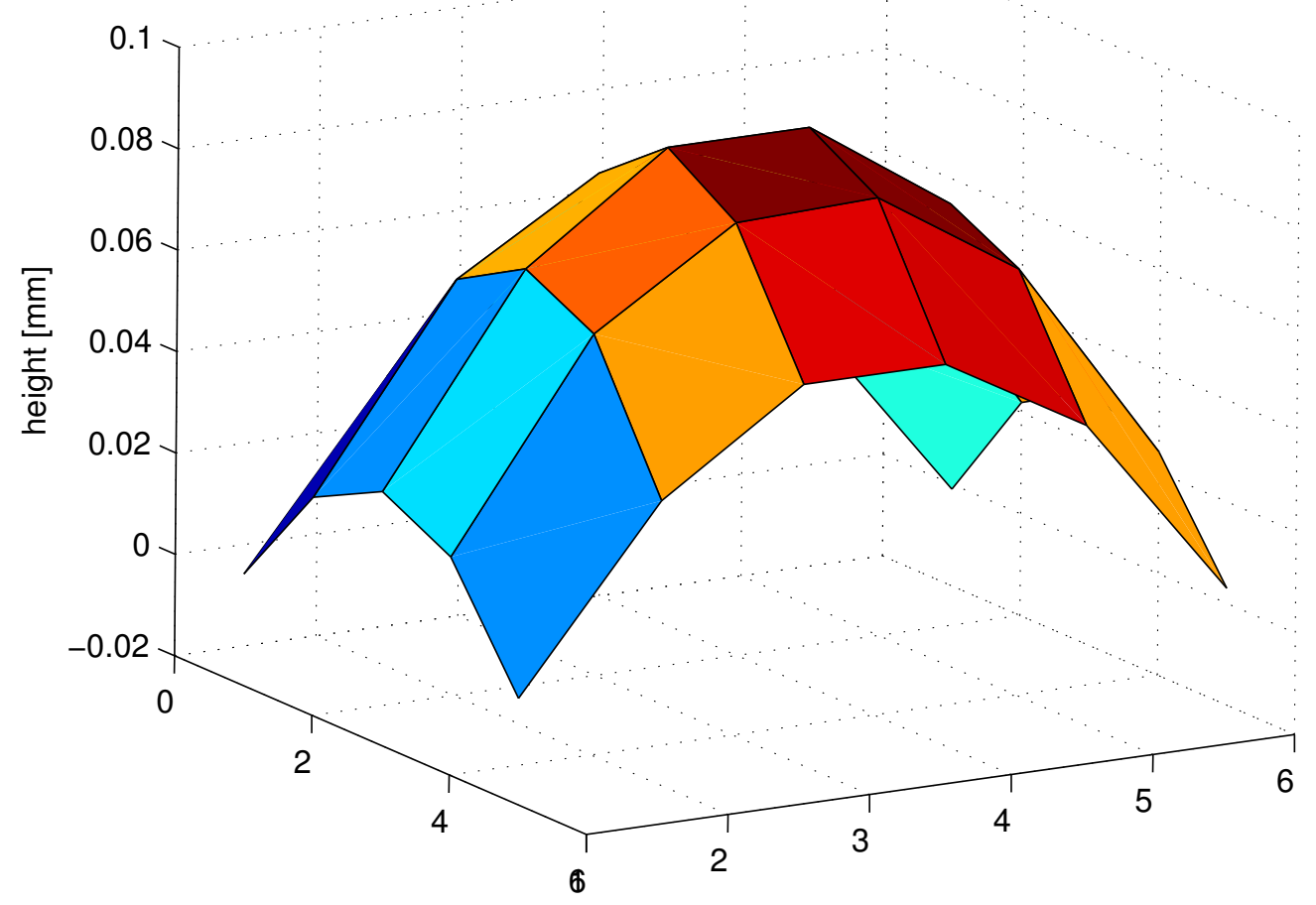




\section{Other tests}

- Coupling capacitances 


\section{Other tests}

- Coupling capacitances

- Other features of leakage currents: 


\section{Other tests}

- Coupling capacitances

- Other features of leakage currents:

- Dependence on mechanical strain 


\section{Other tests}

- Coupling capacitances

- Other features of leakage currents:

- Dependence on mechanical strain

- Reproducibility 


\section{Other tests}

- Coupling capacitances

- Other features of leakage currents:

- Dependence on mechanical strain

- Reproducibility

- Stability over $\sim 24 \mathrm{~h}$ 


\section{Other tests}

- Coupling capacitances

- Other features of leakage currents:

- Dependence mechanical strain

- Reproducibility

- Stability over $\sim 24 \mathrm{~h}$

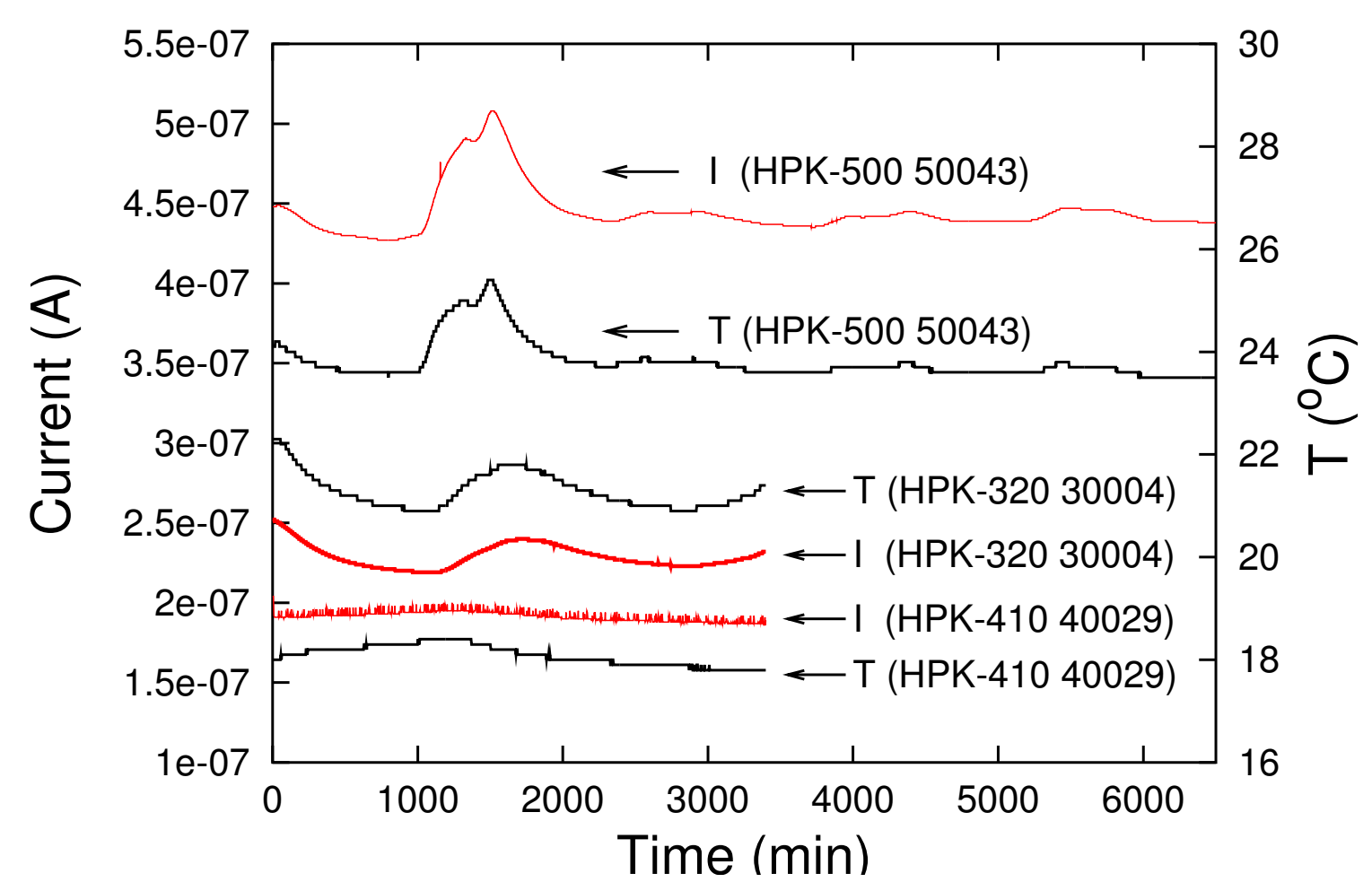




\section{Overview}

- LHCb spectrometer and the Silicon Tracker

- Sensor quality assurance program and results on first sensors

- Irradiation tests with IT prototype sensors

- Summary 


\section{Irradiated Sensors}

- 3 IT Multi-Geometry prototype sensors (5 regions; same thickness, dimensions and material characteristics as HPK-320) 


\section{Irradiated Sensors}

- 3 IT Multi-Geometry prototype sensors (5 regions; same thickness, dimensions and material characteristics as HPK-320)

- 1 CMS-OB2 test-structure (monitor diode, mini-detector, isolated elements of strips, polysilicon, coupling capacitances,...) 


\section{Irradiation}

- T7 irradiation facility, at PS at CERN 


\section{Irradiation}

- T7 irradiation facility, at PS at CERN

- $24 \mathrm{GeV} / \mathrm{c}$ protons; hardness $k=0.6$ 


\section{Irradiation}

- T7 irradiation facility, at PS at CERN

- $24 \mathrm{GeV} / \mathrm{c}$ protons; hardness $k=0.6$

- Fluences:

- One sensor: $1.9 \times 10^{13} \mathrm{p} / \mathrm{cm}^{2}(\sim 7$ years innermost IT $)$

- Remaining: $6.3 \times 10^{13} \mathrm{p} / \mathrm{cm}^{2}(\sim 20$ years innermost IT) 


\section{Irradiation}

- T7 irradiation facility, at PS at CERN

- $24 \mathrm{GeV} / \mathrm{c}$ protons; hardness $k=0.6$

- Fluences:

- One sensor: $1.9 \times 10^{13} \mathrm{p} / \mathrm{cm}^{2}(\sim 7$ years innermost IT $)$

- Remaining: $6.3 \times 10^{13} \mathrm{p} / \mathrm{cm}^{2}(\sim 20$ years innermost IT $)$

- Proton fluences from aluminium foils activation measurements 


\section{Irradiation}

- T7 irradiation facility, at PS at CERN

- $24 \mathrm{GeV} / \mathrm{c}$ protons; hardness $k=0.6$

- Fluences:

- One sensor: $1.9 \times 10^{13} \mathrm{p} / \mathrm{cm}^{2}(\sim 7$ years innermost IT $)$

- Remaining: $6.3 \times 10^{13} \mathrm{p} / \mathrm{cm}^{2}(\sim 20$ years innermost IT $)$

- Proton fluences from aluminium foils activation measurements

- Annealing of $80 \mathrm{~min}$ at $60^{\circ} \mathrm{C}$ 


\section{Electrical characterization}

- Performed at room temperature; between measurements, $-20^{\circ} \mathrm{C}$ 


\section{Electrical characterization}

- Performed at room temperature; between measurements, $-20^{\circ} \mathrm{C}$

- Measured:

- full depletion voltages

- leakage currents $\longrightarrow$ current related damage constant $\alpha$

- AC- and DC- strip tests: strip capacitances, inter-strip capacitances, coupling capacitances, strip currents 


\section{Full depletion voltages}

- Extracted from total sensor capacitance

\begin{tabular}{lccc} 
Sensor & Fluence $\left(\mathrm{p} / \mathrm{cm}^{-2}\right)$ & Vdepl $(\mathrm{V})$ before & Vdepl $(\mathrm{V})$ after \\
\hline LHCb 5 & $1.9 \times 10^{13}$ & 55 & 40 \\
\hline LHCb 8 & $6.3 \times 10^{13}$ & 55 & 130 \\
\hline LHCb 1 & $6.3 \times 10^{13}$ & 55 & 130 \\
\hline Diode & $6.3 \times 10^{13}$ & 120 & 115 \\
\hline
\end{tabular}




\section{Full depletion voltages}

- Extracted from total sensor capacitance

\begin{tabular}{lccc} 
Sensor & Fluence $\left(\mathrm{p} / \mathrm{cm}^{-2}\right)$ & Vdepl $(\mathrm{V})$ before & Vdepl $(\mathrm{V})$ after \\
\hline LHCb 5 & $1.9 \times 10^{13}$ & 55 & 40 \\
\hline LHCb 8 & $6.3 \times 10^{13}$ & 55 & 130 \\
\hline LHCb 1 & $6.3 \times 10^{13}$ & 55 & 130 \\
\hline Diode & $6.3 \times 10^{13}$ & 120 & 115 \\
\hline
\end{tabular}

$\Rightarrow$ After lower fluence, depletion voltage lower than initial 


\section{Leakage currents}

- After irradiation $\Delta I=\alpha V \phi$ 


\section{Leakage currents}

- After irradiation $\Delta I=\alpha V \phi$

- Calculate the current related damage constant $\alpha$ 


\section{Leakage currents}

- After irradiation $\Delta I=\alpha V \phi$

- Calculate the current related damage constant $\alpha$

- Use $I$ at $V_{\text {bias }}=V_{\text {depl., }}$, normalized to $T=20^{\circ} \mathrm{C}$ 


\section{Leakage currents}

- After irradiation $\Delta I=\alpha V \phi$

- Calculate the current related damage constant $\alpha$

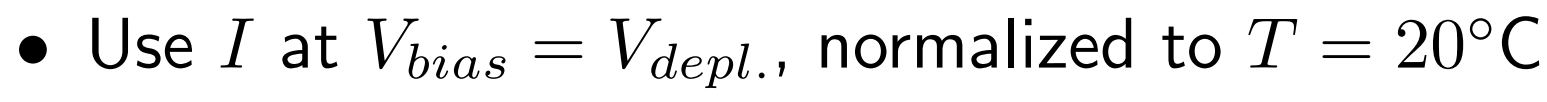

- Obtained for $24 \mathrm{GeV}$ proton, $\bar{\alpha}=2.78 \times 10^{-17} \mathrm{~A} / \mathrm{cm}$ 


\section{Leakage currents}

- After irradiation $\Delta I=\alpha V \phi$

- Calculate the current related damage constant $\alpha$

- Use $I$ at $V_{\text {bias }}=V_{\text {depl. }}$, normalized to $T=20^{\circ} \mathrm{C}$

- Obtained for $24 \mathrm{GeV}$ proton, $\bar{\alpha}=2.78 \times 10^{-17} \mathrm{~A} / \mathrm{cm}$

- Normalizing fluence to equivalent $1 \mathrm{MeV} \mathrm{n}, \rightarrow$ hardness factor $k_{\alpha}=0.61$ 


\section{Strip tests}

- total strip capacitances

- inter-strip capacitances

- coupling capacitances

$\longrightarrow$ essentially unchanged after irradiation 


\section{Overview}

- LHCb spectrometer and the Silicon Tracker

- Sensor quality assurance program and results on first sensors

- Irradiation tests with IT prototype sensors

- Summary 


\section{Summary}

- LHCb Silicon Tracker uses silicon micro-strip detectors

- $200 \mu \mathrm{m}$ pitch

- readout strips up to $38 \mathrm{~cm}$ in length 


\section{Summary}

- LHCb Silicon Tracker uses silicon micro-strip detectors

- $200 \mu \mathrm{m}$ pitch

- readout strips up to $38 \mathrm{~cm}$ in length

- Presented QA program

- results on first sensors very satisfactory

- low leakage currents; electrical parameters within specification 


\section{Summary}

- LHCb Silicon Tracker uses silicon micro-strip detectors

$-\sim 200 \mu \mathrm{m}$ pitch

- readout strips up to $38 \mathrm{~cm}$ in length

- Presented QA program

- results on first sensors very satisfactory

- low leakage currents; electrical parameters within specification

- Performed irradiation on IT prototype sensors with $24 \mathrm{GeV} / \mathrm{c}$ protons

- depletion at $50 \mathrm{~V}$ after equivalent to $7 \mathrm{LHCb}$ years in IT

- current related damage constant $\alpha=2.78 \times 10^{-17} \mathrm{~A} / \mathrm{cm}$, hardness factor $k_{\alpha}=0.61$

- strip capacitances, coupling capacitances: unchanged after irradiation 


\section{Back-slides}




\section{Reproducibility IV curves}

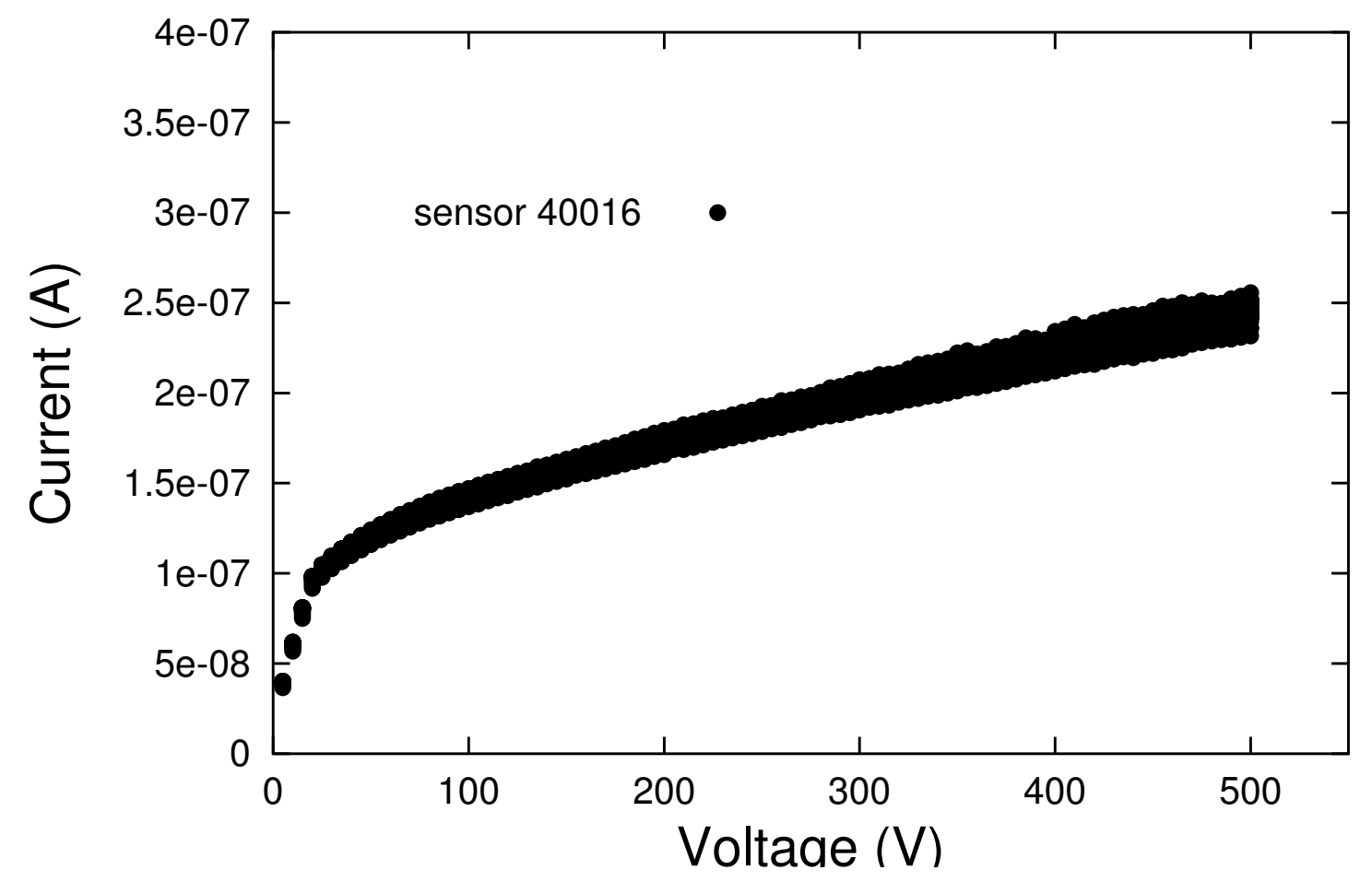




\section{No chuck vacuum effect}

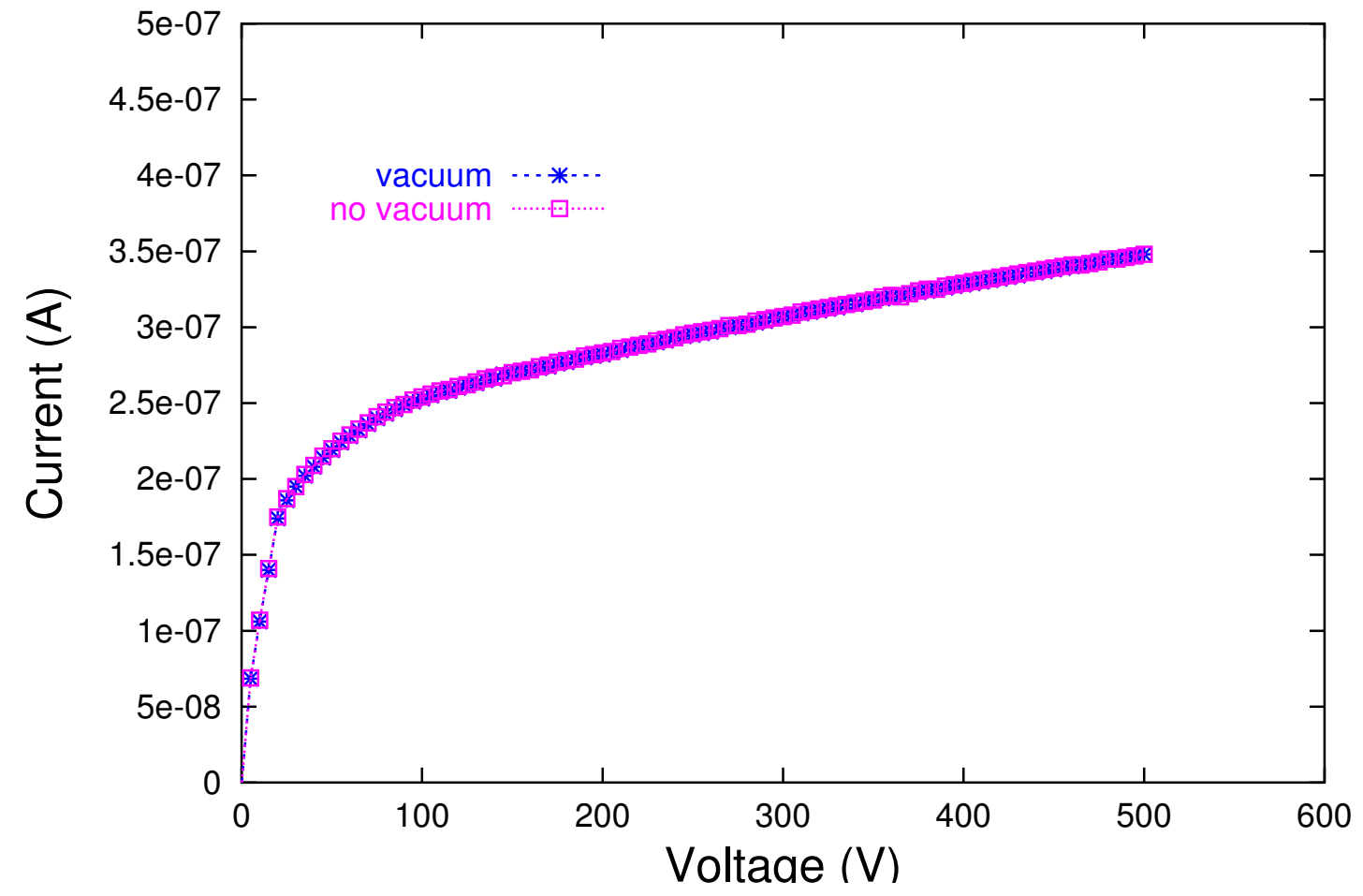




\section{Depletion voltages vs. Hamburg model}

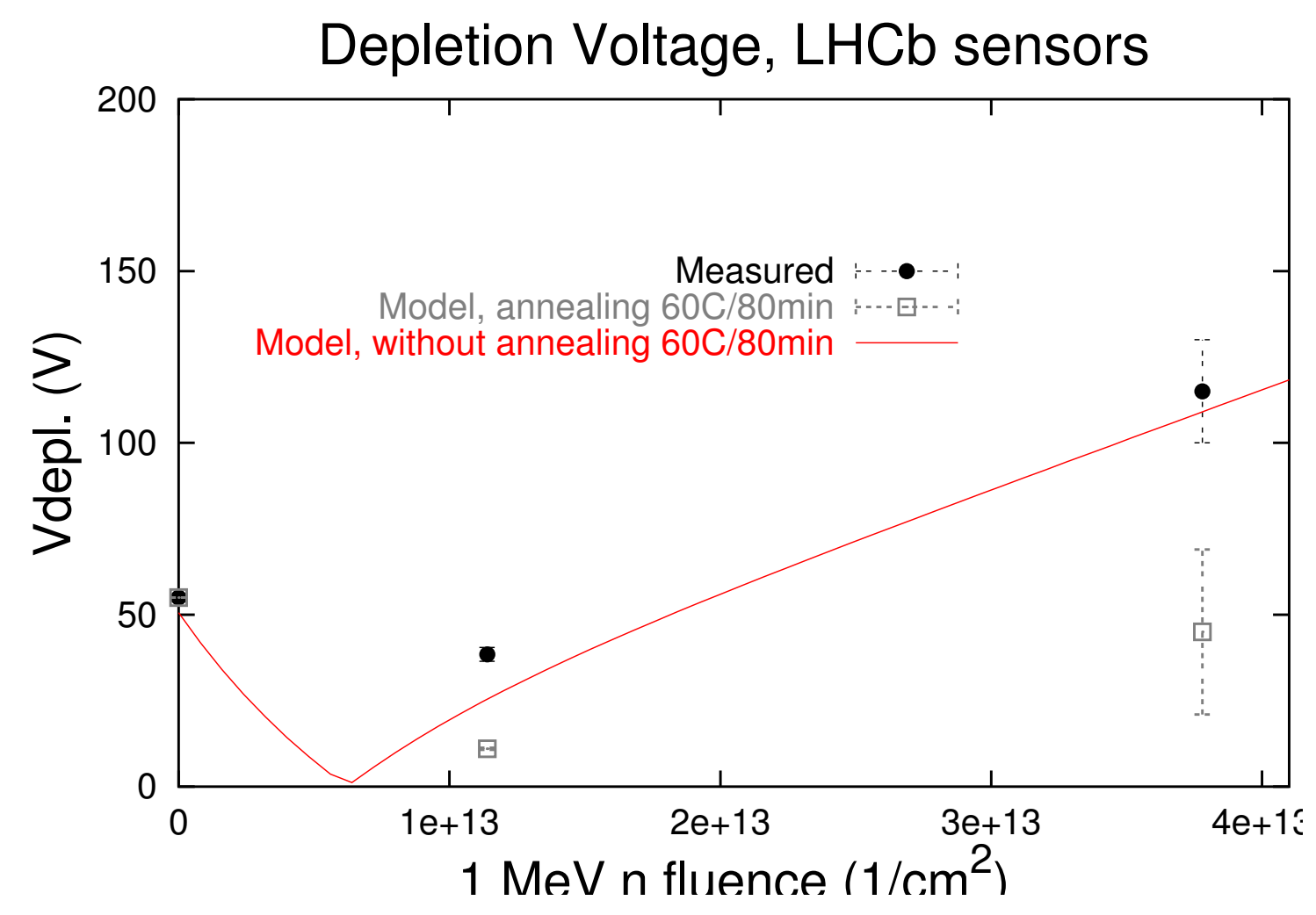




\section{I vs T}

Leakage current:

$$
I(T)=I\left(T_{m}\right)\left(\frac{T}{T_{m}}\right)^{2} \exp \left(-\frac{E_{g}}{2 k_{B}}\left\{\frac{1}{T}-\frac{1}{T_{m}}\right\}\right)
$$

- $E_{g}=1.12 \mathrm{eV}$ band gap energy in silicon at room temperature

- $k_{B}$ Boltzmann constant

- $T$ and $T_{m}$ in $\mathrm{K}$

- relation true if current caused by generation current in the bulk material (case after irradiation) 


\section{References}

- LHCb Technical Design Report, Reoptimized Detector Design and Performance. CERN/LHCC 2003-030.

- LHCb Inner Tracker Technical Design Report. CERN/LHCC 2002-029.

- O. Steinkamp. Silicon strip detectors for the LHCb experiment. Nucl. Instr. and Meth. A 541 (2005) 83.

- F. Lehner, C. Lois and H. Voss. Measurements on irradiated silicon sensor prototypes for the Inner Tracker of LHCb. LHCb note 2004-104.

- G. Baumann et al., Pre-series Sensor Qualification for the Inner Tracker of $\mathrm{LHCb}$. $\mathrm{LHCb}$ note in preparation. 


\section{Table of Contents}

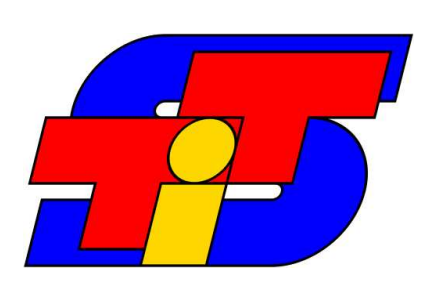

overview Ihcb tt it sensors sensors visual IV IV cV metrology otherTests irrad irradII irradIII summary reproducibility noChuckVacEffect HamburgModel IvsT 